\title{
Control Concurrency Type Code
}

National Cancer Institute

\section{Source}

National Cancer Institute. Control Concurrency Type Code. NCI Thesaurus. Code C93543.

A coded value specifying the relationship between the study control and an entity or event. 